\title{
CURSO CLÍNICO Y RESULTADOS A LARGO PLAZO EN EL TRATAMIENTO COGNITIVO-COMPORTAMENTAL DEL TRASTORNO POR ANGUSTIA CON AGORAFOBIA. ${ }^{1}$
}

\author{
Rafael Ballester Arnal² \\ Cristina Botella Arbona \\ Universitat Jaume I (Castelló)
}

\begin{abstract}
RESUMEN
El tratamiento cognitivo-comportamental se ha constituido como la alternativa terapéutica de elección para el trastorno por angustia. Sin embargo, aunque son numerosos los estudios que demuestran la eficacia terapéutica de este tipo de acercamientos, disponemos de pocos datos que indiquen sus efectos a largo plazo. Pocos estudios presentan seguimientos de los pacientes más allá de un año después de ser retirado el tratamiento. En este trabajo se presenta el procedimiento y resultados a corto y largo plazo (con un seguimiento de 5 años) de la aplicación de un programa cognitivo-comportamental para un caso de trastorno por angustia con agorafobia.
\end{abstract}

PALABRAS CLAVE: Trastorno por angustia, agorafobia, tratamiento cognitivocomportamental, eficacia terapéutica, resultados a largo plazo.

\begin{abstract}
Cognitive-behavioural treatment has been established as the therapeutic alternative of choice for panic disorder. Nevertheless, although there are many studies that evidence the therapeutic effectiveness of these approaches, we have not much information about long-term outcome. There are few studies presenting more than one year follow-up after treatment. At this paper, process, short-term and long-term outcomes (with a five year follow-up) of a cognitive-behavioural programme for a panic disorder with agoraphobia case are presented.
\end{abstract}

KEY WORDS: Panic disorder, agoraphobia, cognitive-behavioural treatment, therapeutic effectiveness, long-term outcome.

\footnotetext{
${ }^{1}$ Este trabajo ha sido posible gracias a la ayuda de Investigación número PS92-0108 concedida por la Dirección General de Investigación Científica y Técnica (DGICYT) del Ministerio de Educación y Ciencia.

${ }^{2}$ Correspondencia: R. Ballester Arnal, Departamento de Psicologia Básica, Clínica y Psicobiologla. Universitat Jaume I. Campus de Borriol. 12080-Castelló
} 


\section{INTRODUCCIÓN}

En los años 80, comenzaron a cobrar importancia los modelos que intentaban explicar el trastorno por angustia desde planteamientos psicológicos. Desde entonces, cada vez han sido más numerosos los estudios dirigidos al análisis de los factores psicológicos que pudieran estar implicados en este problema, así como las estrategias a utilizar para su tratamiento. Hasta el momento, los enfoques terapéuticos más relevantes se han centrado en la reatribución de las sensaciones corporales de pánico (Beck, Emery y Greenberg, 1985; Beck, 1988; Clark y Salkovskis, 1987; Salkovskis, Clark y Hackman, 1991), el entrenamiento en una respiración opuesta a la hiperventilación (Lum, 1976, 1981; Ley, 1985, $1987,1988)$, y la exposición a estímulos internos y externos (Barlow, 1988; Barlow y Cerny, 1988; Marks, 1987). Afortunadamente, ya tenemos datos suficientes para defender la eficacia terapéutica de estas alternativas de tratamiento. Estos datos confirman que, en general, las estrategias cognitivo-comportamentales constituyen el tratamiento de elección para el trastorno por angustia, lo que se justifica por sus altas tasas de éxito $(80 \%)$, sus bajas tasas de abandono, su capacidad de generalización a otros problemas no específicamente tratados y la estabilidad y consistencia de los logros conseguidos con la terapia (Barlow, 1988; Clum, 1989; Michelson y Marchione, 1991; Clum, Clum y Surls, 1993; Margraf, Barlow, Clark y Telch, 1993).

Sin embargo, aunque son numerosos los estudios que demuestran la eficacia terapéutica de este tipo de acercamientos, disponemos de pocos datos que indiquen sus efectos a largo plazo. Pocos trabajos presentan seguimientos de los pacientes más allá de un año después de ser retirado el tratamiento. Tampoco sabemos con precisión cuáles son los factores predictores de una u otra evolución en un determinado paciente, y mucho menos qué elementos terapéuticos habría que integrar en el tratamiento de un determinado paciente con determinadas características clínicas para favorecer el mantenimiento del cambio que se pretende lograr. En el presente trabajo se presenta el procedimiento terapéutico seguido y los resultados a corto y largo plazo (con un seguimiento de 5 años) en un caso de trastomo por angustia con agorafobia, como una aportación a la importante problemática de cómo lograr y mantener el cambio en terapia.

\section{PROCEDIMIENTO}

\subsection{Características personales del paciente}

J.V es un varón de 35 años de edad, casado y con dos hijos de 7 y 2 años. Alcanzó el grado escolar de Bachiller Superior y en el momento de acudir a consulta trabaja como agente comercial de una importante firma. Desde un punto de vista general, los datos personales de J.V. revelan que éste mantiene poco contacto con sus padres. La relación con su padre, con un nivel cultural de estudios primarios es calificada por el paciente como normal. No fue ni muy afectivo con sus hijos, ni tampoco muy autoritario; dejaba a sus hijos un margen de autonomia bastante amplio; por lo demás, había poca comunicación entre ellos. Por lo que respecta a su madre, de idéntico nivel cultural y de carácter autoritario y rigido, el paciente la recuerda lamentándose continuamente por su vida (ella aspiraba a una vida mejor) y considera que supo contagiar esa infelicidad a los demás miembros de la familia. Bajo el punto de vista de J.V., esa forma de comportarse dió lugar a una mala relación entre sus padres. Su madre se hacia la mártir, culpando a su marido de su situación. En la actualidad, la madre de J.V. no conoce su problema, él prefiere no contárselo. Por su parte, la relación con su único hermano, cuatro años mayor que J.V. tampoco ha sido buena desde que eran pequeños. El paciente informa acerca de sentimientos de celos por parte del hermano desde siempre, derivados entre otras cosas de la mayor facilidad que J.V. tenía para los estudios. Ahora, prácticamente no se relacionan.

En cuanto a su familia nuclear, J.V. vive desde hace 12 años con su mujer que tiene su misma edad. A pesar de que ésta constituye la única persona capaz de tranquilizar al paciente en su problema y un punto de apoyo muy importante en su vida, la relación con ella ha pasado por algunas dificultades notables. La comunicación entre ambos es bastante buena. Sin embargo, el paciente considera que en su relación falta afectividad y que las relacio- 
nes sexuales no son del todo satisfactorias. La actitud del paciente hacia el sexo es positiva y únicamente refiere problemas de dispareunia en ambos cónyuges antes y al poco tiempo de casarse. Por lo que respecta a las crisis del paciente, su mujer no les resta importancia, pero considera que él no hace todo lo posible por ponerse bien y que ella no se siente capaz de ayudarle. J.V. afirma que su mujer, en ocasiones, muestra estar cansada por el problema de él. Por lo que respecta a sus dos hijos, no parecen haber problemas importantes que merezcan ser mencionados; más bien al contrario, el paciente mantiene muy buena relación con ellos, participando activa y entregadamente en su crianza.

Por lo que se refiere a su vida social, J.V. la califica como moderada. Tiene amigos, uno de ellos bastante íntimo, con los que se reúne algunos fines de semana y, por lo demás, no dice presentar ninguna dificultad en las relaciones sociales con personas de igual y distinto sexo. No tiene ninguna afición en particular. Únicamente con anterioridad a su problema jugaba al frontón, actividad que abandonó, entre otras cosas, por el miedo a las crisis de angustia. En lo que respecta a su actividad laboral como agente comercial, el paciente informa sentirse satisfecho en líneas generales, considera que su trabajo está de acuerdo con su preparación y además le permite no tener problemas económicos, aunque en el futuro aspiraría a trabajar para él mismo.

Por último, J.V. aseguró en la entrevista no haber padecido ninguna enfermedad grave, ni intervenciones quirúrgicas, a excepción de una operación en el intestino dos años antes de acudir a consulta. El embarazo y parto de su madre sucedieron sin dificultades. Informó de dos accidentes de automóvil algo aparatosos, que no produjeron ninguna lesión en el paciente. Por lo demás, no aparecen problemas relacionados con el sueño y su apetito es calificado como normal.

\subsection{Historia del problema}

El paciente acude en Octubre de 1990 a la consulta del Servicio de Asistencia Psicológica para Problemas de Ansiedad creado en el Departamento de Personalidad, Evaluación y Tratamientos Psicológico de la Universidad de Valencia. En la entrevista de recepción, el mismo paciente refiere sufrir trastorno por angustia con agorafobia, según le han asegurado distintos especialistas a los que ha visitado hasta la fecha. El problema comenzó 11 años antes (cuando J.V. tenía 24 años), momento en que se produjo su primera crisis. El dia anterior de la crisis, habian intervenido quirúrgicamente a su mujer y por alguna complicación, la operación habia tardado más de lo previsto, lo que supuso un gran estrés para el paciente. Al dia siguiente y sin dormir, J.V. fue a trabajar. Tras finalizar su jornada, prácticamente sin comer, acudió al hospital a ver a su mujer, y en el trayecto, comenzó a sentir una sudoración intensa, palpitaciones, taquicardia y una fuerte sensación de inestabilidad (el paciente lo describe con gestos muy expresivos que denotan la angustia del momento). Al tomarse el pulso, notó que tenia 160 pulsaciones por minuto, lo que todavia le asustó más, hasta el punto que creyó que iba a morir. Aunque no le habia sido prescrito por ningún profesional, tomó un valium (diazepan) y se le pasaron los sintomas. Antes de ese suceso, el paciente nunca habia tenido miedo de sus propias sensaciones corporales, pero desde entonces, comenzó a desarrollar una importante preocupación somática. En un principio, el curso del problema era creciente, esto es, J.V. tenía crisis cada vez con mayor frecuencia (más de una al día). Más tarde, el problema se estabilizó e incluso remitió, llegando a estar dos años completos sin ninguna crisis de angustia, si bien es cierto, que durante ese periodo J.V. desarrolló una evitación cada vez mayor de numerosas situaciones y actividades por miedo a experimentar una crisis. Asímismo padecía algunos sintomas de ansiedad (especialmente, mareos) de manera constante, que no llegaban a culminar en una crisis. La vida del J.V. se iba autolimitando cada vez más hasta niveles importantes, como por ejemplo, la evitación de emociones fuertes que hicieran experimentar al paciente una intensa alegria, tristeza, enfado o nerviosismo, movimientos corporales rápidos al andar e incluso al gesticular, ir solo a cualquier lugar alejado de su casa, coger el coche para desplazarse, viajar, ir a un restaurante o a unos grandes al-macenes, hacer colas, estar donde hubiera mucha gente, subir a atracciones de 
feria, tomar cualquier tipo de medicamento (especialmente psicofármacos), utilizar transportes públicos, tráfico denso, ascensores desconocidos, entrar en un hospital, hacerse un análisis de sangre, practicar cualquier deporte, pasar calor, beber alcohol, café o cocacola, hacer esfuerzos físicos, ir al cine, ir solo con sus hijos por si le pasaba algo y no podia atenderlos y ducharse estando solo en casa. El paciente se mantuvo en el frágil equilibrio que otorga una evitación de esta magnitud hasta un año antes de acudir a nuestra consulta, como explicaremos seguidamente.

Durante todo este tiempo, el paciente llevó a cabo un intenso peregrinaje por todo tipo de especialistas. En un principio, hace 11 años, visitó un médico de medicina general que explicó a J.V., según sus propias palabras, que este problema le ocurría por "no estar de acuerdo con la vida que llevaba". A continuación, la preocupación por padecer una enfermedad que explicase sus crisis, le condujo a la consulta de varios cardiólogos y neurólogos. Una vez descartado cualquier trastorno orgánico como causante de sus crisis, el paciente fue a un psiquiatra que le daba "terapia de apoyo y pastillas". Parece ser que J.V. salia tranquilizado de la consulta, pero, al momento volvía a encontrarse mal. Entonces, le aconsejaron ir a un psicoanalista con el que estuvo tres años a razón de una o dos sesiones semanales. Según el psicoanalista, J.V. padecia en realidad un problema sexual, sus crisis eran la expresión del miedo y la culpabilidad experimentada por el paciente en determinadas relaciones sexuales que tuvo poco antes de comenzar las crisis. Sin embargo, al paciente no le convenció esta explicación, además de "estar harto de que el terapeuta no contestase mis preguntas, o las contestara con más preguntas". Seguidamente, acudió a un psicólogo que le aplicó durante un año análisis transaccional y que, según él, no fue capaz de ayudarle en nada. Estuvo un tiempo desanimado, sin decidirse a ir a ningún profesional, y finalmente, un año antes de buscar nuestra ayuda, fue a un médico internista, que le dijo por primera vez que lo que él tenia se denominaba trastorno por angustia. Le prescribió anafranil y, posteriormente, tranquimazin, que tuvo que dejar de tomar porque también le daba miedo y le desencadenaba crisis el hecho de pensar en la reacción que podian causarle. Ante esta situación, el médico le dijo que resultaba necesario que él se expusiera a las situaciones temidas para poder superar su problema. Sin embargo, J.V. no se sentia capaz de en-frentarse a las situaciones que le podían desen-cadenar una crisis de angustia, porque era "como si uno buscase a propósito sentir que se está muriendo, a pesar de que le asegurasen que no le podia ocurrir nada". Un año después el paciente acudió a nuestro Servicio de Asistencia Psicológica.

\subsection{Diagnóstico}

Para el diagnóstico del paciente se utilizó la Entrevista estructurada SCID-P de Spitzer y Williams (1987) con los criterios del DSMIII-R. J.V. cumplia los criterios para el diagnóstico de Trastorno por angustia con agorafobia con un grado "moderado" de gravedad como se califica a la evitación que permite al individuo salir solo de casa, sin desplazarse más allá de unos kilómetros sin compañía. El terapeuta, sin embargo, teniendo en cuenta la globalidad del caso, especialmente el amplio espectro de actividades evitadas (más allá de las situaciones agorafóbicas recogidas en los criterios del DSM-III-R), la antigüedad del problema y otras variables clínicas como el grado de preocupación somática, y de autoatención corporal, valoró el caso como grave. Por lo demás, descartado el papel causal de alguna lesión orgánica a partir de la historia clínica, se realizó el diagnóstico diferencial respectó de la ansiedad generalizada y la hipocondría.

\subsection{Evaluación}

Como resulta habitual en nuestro Servicio de Asistencia Psicológica, una vez delimitado el diagnóstico del paciente, se llevó a cabo una amplia evaluación en la que se tomaron en consideración no sólo las variables directamente relacionadas con la manifestación de las crisis de angustia y la agorafobia, sino también otras variables de relevancia clínica, como el neuroticismo, la ansiedad-estado y ansiedad-rasgo, el grado de depresión, la conducta de enfermedad (hipocondria, conciencia corporal privada...) y otras variables 
que tienen que ver con la valoración que hace el paciente de su malestar subjetivo, la interferencia del problema en su vida y la valoración de paciente y terapeuta de la evolución del problema.

En la tabla número 1, presentamos las variables evaluadas, asi como los instrumentos utilizados.
De entre las variables mencionadas en la tabla número 1 , sólo eran evaluadas semanalmente las referidas a la frecuencia, intensidad y sintomas de las crisis (Diario de Pánico), el grado de creencia en las interpretaciones catastróficas (en el caso de que se hubiera producido alguna crisis en la semana), la evitación/ temor agorafóbicos (EET), la ansiedad gene

\section{Tabla 1.- Variables evaluadas $\mathrm{e}$ instrumentos utilizados}

- Anamnesis: Adaptación de la entrevista semiestructurada de Lazarus

- Variables directamente relacionadas con las crisis de angustia como la frecuencia de crisis, su intensidad y síntomas con el Diario de Pánico (adaptado de Clark y Salkovskis, 1987); las cogniciones catastróficas con el Cuestionario de Pensamientos Distorsionados de Beck (CDQ) (Beck, 1988); y el grado de creencia en los principales miedos que el paciente tenia en sus crisis, a saber, miedo a sufrir un ataque cerebral, miedo a padecer un infarto y miedo a desmayarse, tanto en el momento de la crisis como en la sesión terapéutica, evaluados a través de escalas de 0 a 100.

- Variables relacionadas con la evitación agorafóbica. Grado de evitación y temor (de 0 a 10) hacia determinadas situaciones o actividades a través de la Escala de Evitación-temor agorafóbicos (EET) adaptada a Marks y cols. (1975). En el caso de nuestro paciente, de entre las actividades evitadas, se seleccionaron como conductas-objetivo por su significación para el paciente las siguientes: pasear, ir al cine, coger un autobús e ir a comprar a un centro comercial. Las puntuaciones que se recogieron a través de la escala mencionada se refieren a la evitación/temor de realización de estas actividades y situaciones, solo, ya que las puntuaciones serían algo menos en caso de referirse al afrontamiento de las mismas situaciones estando acompañado.

- Variables de personalidad, variables clínicas generales y de conducta de enfermedad, tales como el grado de neuroticismo (EPI de Eysenck y Eysenck, 1978); la ansiedad-estado y ansiedad-rasgo (STAl de Spielberger, Gorsuch y Lushene, 1970); el grado de ansiedad semanal evaluado a través del BAI (Inventario de Ansiedad de Beck) y de la Escala Subjetiva de Ansiedad (ESA) de 0 a 10; el grado de depresión (BDI de Beck, 1979, versión de Conde y Franch, 1984); convicción de enfermedad, problemas afectivos y grado de hipocondría (Cuestionario de Conducta de Enfermedad, IBQ, de Pilowsky y Spence, 1983, adaptado por Ballester y Botella, 1993a); autoconciencia privada - grado de atención difusa dirigida hacía uno mismo (Escala SCS de Autoconciencia Privada y Pública de Fenigstein, Scheier y Buss, 1975) (adaptado por Ballester, 1992); y la conciencia corporal privada 0 grado de focalización atencional hacia el funcionamiento corporal (Escala BCS de Con ciencia Corporal Privada y Pública de Miller, Murphy y Buss, 1981) (adaptado por Ballester, 1992) ${ }^{3}$

- Otras variables clínicas, como la valoración del paciente acerca del malestar generado por el trastorno (Escala ESM de 0 a 10), el grado en que éste interfiere en su vida (Escala global de 0 a 5 del Cuestionario de Adaptación, CA de Echeburúa y De Corral, 1987); la valoración que el paciente hace de su mejoria o no (Escala S-6 de Marks de 1 a 7 donde 1 es "muchísimo mejor", 2 "bastante mejor", 3 "un poco mejor", 4 "sin cambios", 5 "un poco peor", 6 "bastante peor" y 7 "muchísimo peor"), asi como, la valoración del terapeuta del estado y evolución del paciente (Escala S-5 de Marks, de 1 a 6 donde 1 es "normal", 2 "ligeramente perturbado", 3 "moderadamente perturbado", 4 "bastante perturbado", 5 "gravemente perturbado" y 6 "muy gravemente perturbado").

${ }^{3}$ El IBQ de Pilowsky y Spence (1983) incluye, además de los factores citados, los de Percepción Psicológica/somática de la enfermedad, Inhibición afectiva, Negación e Irritabilidad. La Escala de Autoconciencia Privada y Pública de Fenigstein y cols. (1975) incluye, asimismo, además del factor de Autoconciencia Privada, los de Autoconciencia Pública y Ansiedad social. Por último, la Escala de Conciencia Corporal Privada y Pública de Miller y cols. (1981) contempla, además del factor de Conciencia Corporal Privada, los de Conciencia Corporal Pública y Competencia Corporal. La razón de haber seleccionado los factores mencionados radica en la significación clinica que estos factores tienen en el trastorno por angustia, así como en este caso en concreto. 
ral en una escala de 0 a 10 (ESA) y la valoración de paciente y terapeuta del grado de mejoría desde el comienzo de la terapia (S$6,5-5)$. El resto de variables eran evaluadas antes y después del tratamiento (la evaluación inicial se llevó a cabo en dos sesiones en dos semanas consecutivas) y en los seguimientos realizados a los $3,6,12$ meses y a los 5 años de finalizar la terapia.

Aunque presentaremos en el epígrafe de resultados (tabla número 6) todos los datos referidos a la evaluación clínica, a nivel descriptivo podemos señalar que el paciente acudió a nosotros valorando su problema como muy grave ( 9 en una escala de 0 a 10) y con muy bajas expectativas de cambio ( 2 en una escala de 0 a 10). En el momento de buscar nuestra ayuda, las crisis se producian con una frecuencia de, aproximadamente, una vez por semana. Las sensaciones corporales que solian acompañar a las crisis eran: falta de aire, mareos, temblor, sudoración, sensación de atragantamiento, hormigueo y sobre todo, cierta sensación de entumecimiento en la cabeza (frente) que le hacia pensar que iba a morir de forma fulminante por un ataque cerebral. Otras veces pensaba que le podia dar un infarto 0 , también, desmayarse. Las sensaciones corporales solian alcanzar su punto álgido en apenas unos segundos, aunque en ocasiones, las crisis iban precedidas de una sensación de ansiedad flotante que podía durar todo un dia. En general, solía presentar sintomas de ansiedad continuos, como el mareo, en el periodo entre crisis. Éstas le podian ocurrir en cualquier situación, aunque en algunas, como las descritas anteriormente, la probabilidad era mucho mayor, por lo que mantenia férreamente la evitación de tales situaciones y actividades. Ante las crisis, el paciente solía ir a su casa, buscar compañía, tumbarse sujetándose la cabeza e intentar distraerse. Habia experimentado crisis con cualquier persona, incluidas su mujer, que solía tranquilizarlo, y sus hijos, todavía muy pequeños para entender lo que le estaba sucediendo a su padre. Hay que decir que, precisamente una de las preocupaciones de J.V. en las crisis era disimular ante sus hijos para no preocuparlos y no transmitirles en un futuro su problema. Asimismo, le preocupaba que compañeros o clientes notasen su problema. Como factores relacionados, el paciente informó que aspectos como el calor, la ingesta de cafeina y la hora del día eran capaces de facilitar sus crisis. Por ejemplo, aunque era frecuente que se despertase bien, y no experimentase ansiedad hasta transcurridos unos quince minutos (en los que el paciente focalizaba la atención en sus sensaciones corporales y anticipaba los afrontamientos de situaciones inevitables), en general, las crisis se daban entre las 20 y las 24 horas del dia, es decir, estando en su propia casa.

Por último, decir que, según el paciente, el problema se debía a factores como cierta predisposición genética (su hermano también habia tenido ahogos nocturnos); rigidez y severidad en el ambiente familiar (de pequeño su madre no le dejaba jugar para que todo estuviese en orden); el desacuerdo más o menos inconsciente con lo que habia sido su vida; y la ansiedad que le producía su relación de pareja y los problemas que había tenido en ella. Sin embargo, aunque el paciente comprendia que todos estos factores le produjeran ansiedad, no podia entender que ésta llegase al extremo y la intensidad de las crisis de angustia.

\subsection{Tratamiento}

El tratamiento aplicado a nuestro paciente se realizó en dos fases diferentes:

a) Primera fase. En esta primera fase, se le aplicó un programa cognitivo-comportamental, específicamente desarrollado para el tratamiento del trastorno por angustia. Concretamente se trata de nuestra adaptación del programa de Clark y Salkovskis (1987) que tiene como objetivo principal la identificación y modificación de los pensamientos catastróficos que, según el modelo cognitivo del trastorno por angustia, ocupan un papel central en las crisis (Botella y Ballester, 1991; Ballester y Botella, 1992). El programa incluye también el entrenamiento en un ritmo lento de respiración contrapuesto a la hiperventilación, técnicas de distracción que ayudan a dejar de focalizar la atención en el estimulo temido, así como experimentos conductuales que contribuyen a que el paciente, por si mismo, someta a prueba la validez o certeza de sus creencias. El componente terapéutico de exposición no aparece estructurado o sistematizado en este programa. Sólo se le dan al paciente instrucciones generales acerca de la convenien- 
cia de afrontar las situaciones temidas con el fin de someter a prueba sus creencias y las técnicas de control de la ansiedad aprendidas en la terapia. Todo ello, dentro de un estilo socrático, caracterizado por el empirismo colaborador en el que el terapeuta ayuda al paciente a buscar evidencias a favor o en contra de sus creencias irracionales y mantiene un feedback continuo con él.

El contenido del programa se presenta detalladamente en otros trabajos (Botella y Ballester, 1991; Ballester y Botella, 1992). Por esta razón, nos limitaremos aquí a señalar la agenda terapéutica que se estableció para cada una de las diez sesiones de tratamiento (una por semana de una hora de duración), la cual aparece resumidamente en la tabla número 2 . Puesto que todas las sesiones comenzaban con la revisión de la tarea y el pase de cuestionarios se han omitido estos puntos. b) Segunda fase. La segunda fase del tratamiento se llevó a cabo a los tres años de finalizar el primer tratamiento, cuando el paciente buscó de nuevo nuestra ayuda "porque se encontraba con fuerzas para comenzar a enfrentarse a las situaciones que evitaba". En su opinión, el origen de su motivación era que se habia dado cuenta de que podia controlar sus crisis por primera vez en su vida. Durante tres años no habia tenido crisis espontáneas y deseaba eliminar la ansiedad residual que en ocasiones experimentaba, así como las limitaciones que le imponia su evitación agorafóbica. Esta segunda intervención terapéutica tuvo lugar, pues, entre el seguimiento número 3 y el seguimiento número 4 . Constó de un total de 40 sesiones de una hora de duración mantenidas a lo largo de un intervalo de 15 meses. El objetivo de esta segunda fase

Tabla 2.- Agenda y contenido de las sesiones de terapia en la primera fase

1 - Descripción de la última crisis (Situación, sensaciones corporales, pensamientos y comportamiento).

- Delimitación de otras situaciones en las que han ocurrido crisis.

- Detalle de las situaciones o actividades evitadas por miedo a una crisis de angustia.

- Reacción habitual del paciente y de otras personas ante las crisis.

- Existencia de moduladores de las crisis.

- Comienzo y curso del problema.

- Tratamientos psicológicos y farmacológicos que ha recibido.

- Causa percibida.

- Tarea: Registrar las crisis de la semana.

2 - ¿Qué es la ansiedad?

- Valor adaptativo de la ansiedad.

- Ausencia de consecuencias perjudiciales de la ansiedad para el organismo.

- Manifestaciones de la ansiedad (Triple Sistema de Respuesta).

- Papel central de los pensamientos en el desencadenamiento de la ansiedad.

- Presentación del modelo cognitivo de las crisis de angustia.

- Prueba de hiperventilación.

- El lugar de la hiperventilación en las crisis.

- Tarea: Registrar las interpretaciones catastróficas durante las crisis de la semana, así como el grado de creencia.

3 - Dudas del paciente respecto al modelo cognitivo del trastorno por angustia y el papel de la hiperventilación: J.V. que tiene un buen grado de información, plantea la dificultad de pensar racionalmente en las crisis y la posibilidad de que a largo plazo los sintomas de ansiedad sean nocivos.

- Entrenamiento en respiración lenta (tumbado o sentado).

- Tarea: Práctica de la respiración lenta (2 veces de media hora al dia). 


\section{SESIÓN}

\section{AGENDA}

4 - Problemas con la respiración lenta. J.V. se angustiaba al practicar la respiración lenta. Una prueba demuestra que hacía una espiración tan profunda que le obligaba a tomar aire con urgencia. Además de corregir el error, se realiza un experimento conductual para que el paciente pueda comprobar que es imposible ahogarse por respirar demasiado poco.

- Discusión cognitiva en torno al miedo a la asfixia.

- Entrenamiento en respiración lenta en posición de pie.

- Tarea: Práctica de la respiración lenta de pie.

5 - Problemas aparecidos con la respiración lenta de pie. J.V. plantea que le resulta muy difícil realizar la respiración lenta en plena crisis. Comentamos el momento adecuado de utilizarla y los efectos esperados.

- Discusión cognitiva en torno a la cognición catastrófica: "En cualquier momento puedo caerme y morir de un ataque cerebral" aparecida en algunas crisis.

- Tarea: Practicar respiración lenta y registrar y retar las cogniciones catastróficas en el diario de pánico.

6 - Planteamiento de la necesidad de afrontar las situaciones asociadas a las crisis con el fin de poner a prueba sus creencias y las técnicas aprendidas para combatir la ansiedad.

- Introducción de un cuestionario destinado a evaluar la exactitud en la predicción de las crisis.

- Tarea: Afrontar alguna de las situaciones evitadas.

7 - Discusión cognitiva respecto al miedo al desmayo y respecto a la tendencia a sobrepredecir la ocurrencia, intensidad y miedo de sus crisis.

- Experimento conductual con el fin de enfatizar el papel de la autoatención en las crisis de angustia.

- Entrenamiento en técnicas de distracción.

- Tarea: Practicar las técnicas de distracción y seguir afrontando.

8 - Experimento conductual para generar sensaciones corporales: correr por la Facultad.

- Discusión acerca de la normalidad de experimentar sensaciones corporales ante un esfuerzo físico y cómo la evitación refuerza el miedo y las cogniciones catastróficas.

- Discusión cognitiva de la crisis de la semana: "Voy a morir de un infarto o cuanto menos a desmayarme".

9 - Valoración de la evolución del paciente a lo largo de la terapia.

- Repaso del contenido de las sesiones anteriores

- Atribución de la mejoría por parte del paciente.

- Refuerzo del terapeuta.

- Valoración de la creencia residual en las interpretaciones catastróficas de las sensaciones corporales.

- Expectativas futuras del paciente con respecto a las crisis.

- Conveniencia de generalizar el contenido de la terapia a otras sensaciones corporales distintas a las habituales, que pudieran aparecer en futuras crisis.

- Conveniencia de seguir practicando las técnicas aprendidas a lo largo de la terapia.

- Tarea: Realizar el "examen de las crisis de angustia".

10 - Comentario de las respuestas dadas por el paciente a las cuestiones del examen: a)¿Qué son las crisis de angustia y cómo se producen?; b)Pensamientos que tenia en una crisis, evidencias a favor y en contra, datos de la terapia que han sido especialmente útiles; c) ¿Cómo puedo afrontar una crisis de angustia?.

- Valoración final de la terapia.

- Emplazamiento para el postest y los ulteriores seguimientos.

ya no fue la disminución del número de crisis de angustia, puesto que dicho objetivo ya se había conseguido con el primer tratamiento, sino la mejoría en la evitación/temor agorafó- bicos, la autoatención corporal y el miedo a la enfermedad. Por to que respecta al procedimiento seguido, el contenido de las sesiones incluyó la exposición a estimulos internos y 
externos (un total de 38 sesiones), técnicas de relajación (6 sesiones), discusión cognitiva "focal" (centrada en las crisis) y "no-focal" (centrada en aspectos más generales como el trabajo, las relaciones familiares...) (15 sesiones), instrucciones basadas en los métodos paradójicos (1 sesión), y el énfasis en la autoeficacia percibida ( 2 sesiones). De esta manera, el terapeuta utilizó las estrategias y técnicas necesarias para la intervención en las áreas clínicamente relevantes teniendo en cuenta la evolución del propio paciente. En la tabla número 3 exponemos sucintamente los elementos terapéuticos utilizados, así como el número de sesiones que se dedicó a cada uno de ellos (el orden de enumeración no implica secuencialidad).

La aplicación del tratamiento se llevó a cabo por el primer autor de este artículo, con amplia experiencia en el tratamiento del trastorno por angustia.

\subsection{Resumen de la secuencia en el proceso de evaluación y tratamiento}

Como puede apreciarse en la tabla número 4, el proceso de evaluación e intervención terapéutica siguió los siguientes pasos: tras dos semanas de establecimiento de la línea base, en las que se realizó el diagnóstico y anamnesis del paciente, se aplicó la primera fase de tratamiento en la que se siguió de modo estructurado el programa cognitivo-comportamental descrito con anterioridad, con una duración de 10 sesiones (10 semanas). Una semana después de finalizado el tratamiento se evaluó de nuevo al paciente en todas las variables clínicas (postest). Posteriormente se realizaron tres seguimientos a los 3 meses, 6 meses y 1 año del postest. A los 3 años y medio de la finalización del primer tratamiento se realizó la segunda intervención terapéutica que constó de 40 sesiones a lo largo de 15 meses. Por último, medio año más tarde, esto es, a

Tabla 3.- Contenido de las sesiones en la segunda fase del tratamiento

\begin{tabular}{|l|c|}
\hline Contenido & Sesiones \\
\hline - Exposición "in vivo" a estimulos externos (planificación y revisión). & 19 \\
- Exposición "in vivo" a estímulos internos (hiperventilación). & 13 \\
- Exposición en imaginación a estímulos externos. & 6 \\
- Análisis, explicación y discusión cognitiva a partir de los momentos de & 10 \\
- Tersiedad de la semana.. & 5 \\
- Entrenamiento en relajación muscular. & 6 \\
- Evaluación global de la situación clinica del paciente. & 3 \\
- Repaso y profundización en técnicas de distracción y respiración lenta. & 3 \\
- Apoyo al paciente (enfatizar locus interno para éxitos, autoeficacia percibida). & 2 \\
- Instrucciones basadas en los métodos paradójicos para controlar la ansiedad. & 1 \\
- Análisis de la conducta de enfermedad del paciente (miedo a la enfermedad, & 1 \\
\hline conciencia corporal). & 40 \\
\hline Total de sesiones & \\
\hline
\end{tabular}


Tabla 4.- Curso temporal del procedimiento de evaluación e intervención

\begin{tabular}{|c|c|c|c|c|c|c|c|c|c|}
\hline & & & $\begin{array}{l}\text { Trat.1 } \\
\text { (10 ses.) }\end{array}$ & Postest & Seg.1 & Seg. 2 & Seg. 3 & $\begin{array}{l}\text { Trat.2 } \\
\text { (40 ses.) }\end{array}$ & Seg. 4 \\
\hline Semana & 1 & 2 & $3-12$ & 13 & 3 meses & 6 meses & 1 año & $3,5-4,5$ a & 5 años \\
\hline $\begin{array}{l}\text { SCID-UP } \\
\text { Anamnesis } \\
\text { Diario pán. } \\
\text { CDQ } \\
\text { EET } \\
\text { EPI } \\
\text { STAI } \\
\text { BAI } \\
\text { ESA } \\
\text { BDI } \\
\text { IBQ } \\
\text { SCS } \\
\text { BCS } \\
\text { ESM } \\
\text { CA } \\
\text { Esc. S-6 } \\
\text { EsC. S-5 }\end{array}$ & * & $\begin{array}{l}* \\
* \\
* \\
* \\
* \\
\star \\
\star \\
\star \\
\star \\
\star \\
\star \\
\star \\
\star \\
\star \\
\star \\
\text { * }\end{array}$ & $\begin{array}{l}* \\
* \\
*\end{array}$ & $\begin{array}{l}* \\
\star \\
* \\
\star \\
* \\
* \\
\star \\
\star \\
\star \\
* \\
\star \\
\star \\
\star \\
\star \\
\star\end{array}$ & $\begin{array}{l}* \\
* \\
* \\
* \\
* \\
* \\
* \\
* \\
* \\
* \\
* \\
* \\
* \\
* \\
*\end{array}$ & $\begin{array}{l}\star \\
\star \\
\star \\
\star \\
\star \\
\star \\
\star \\
\star \\
\star \\
\star\end{array}$ & $\begin{array}{l}* \\
\star \\
* \\
\star \\
\star \\
\star \\
\star \\
\star \\
* \\
\star \\
\star \\
\star \\
\star \\
\star \\
\star\end{array}$ & $\begin{array}{l}* \\
*\end{array}$ & $\begin{array}{l}\star \\
\star \\
\star \\
\star \\
\star \\
\star \\
\star \\
\star \\
\star \\
\star \\
\star \\
\star \\
\star \\
\star\end{array}$ \\
\hline
\end{tabular}

los cinco años de finalizada la primera fase de tratamiento, se estableció el cuarto seguimiento en el que se volvieron a evaluar todas las variables.

\section{RESULTADOS}

Con el fin de analizar con detalle la evolución del paciente a lo largo del proceso terapéutico, así como el resultado de la intervención, hemos clasificado las variables en cuatro grupos. Asi en la tabla número 5 , presentamos los datos referidos a las variables directamente relacionadas con las crisis, tales como la frecuencia, intensidad, sensaciones corporales y cogniciones catastróficas presentes en las crisis. En la tabla número 6, exponemos los datos referidos a la evitación y temor agorafóbicos para las cuatro conductas objetivo, a saber, pasear, ir al cine, coger el autobús e ir de compras a un centro comercial. En la tabla número 7 , los datos referidos a las variables de personalidad como el neuroticismo; varia- bles clínicas como la ansiedad estado y rasgo y el grado de depresión; y la conducta de enfermedad del paciente. $Y$, en la tabla número 8 , las variables relacionadas con la valoración global que paciente y terapeuta hacen del estado del paciente y de su grado de mejoria. Por otra parte, para poder apreciar con más claridad la mejoria clínica en algunas variables, presentamos entre paréntesis los porcentajes de mejoria respecto del pretest. Siguiendo la clasificación utilizada en otros trabajos (Visser y Bouman, 1992; Martínez y Botella, en prensa; Ballester, Botella y Carrió, 1995) consideraremos "muy mejorado" al paciente cuando se dé una disminución superior al $70 \%$ en las variables evaluadas; "mejorado" cuando la disminución se sitúa entre el 31 al $69 \%$; y "no mejorado" significativamente cuando la disminución es inferior al $30 \%$. Asímismo en las figuras número 1, 2 y 3 es posible observar de modo gráfico la evolución del paciente en las variables más relevantes. 


\begin{tabular}{|c|c|c|c|c|c|c|c|c|c|}
\hline 苛 & $\begin{array}{l}n \\
0 \\
0 \\
0 \\
0 \\
0\end{array}$ & o & 이 & 0 & 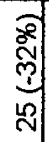 & $1-$ & 10 & 15 & 웅 \\
\hline $\begin{array}{l}3 \\
\text { 岁 }\end{array}$ & 空 & 0 & 이 & 0 & $\frac{a}{5}$ & : 요 & : 유 & $1 \stackrel{n}{\sim}$ & 各 \\
\hline $\begin{array}{l}y \\
\vdots \\
w\end{array}$ & $\underset{\dot{g}}{\dot{g}}$ & o & 이 & o & $\stackrel{\infty}{\mathrm{N}}$ & : 의 & 16 & 1 으 & よ̊ \\
\hline 岕 & $\begin{array}{l}\stackrel{g}{g} \\
\stackrel{\$}{E} \\
m\end{array}$ & 이 & 이 & 0 & 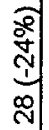 & $\begin{array}{ll}1 & 10\end{array}$ & $1 \mathrm{~m}$ & 160 & 各 \\
\hline $\begin{array}{l}5 \\
0 \\
0 \\
0\end{array}$ & $\stackrel{m}{s}$ & 0 & 이 & 이 & $\begin{array}{l}2 \\
\frac{2}{N} \\
\frac{1}{N}\end{array}$ & $1 \quad n$ & $: m$ & 16 & ڤ̊̊ \\
\hline & $\frac{v}{s}$ & 0 & 이 & 이 & $i$ & 111 & $\begin{array}{ll}1 & 1\end{array}$ & 11 & \\
\hline & $\frac{F}{\infty}$ & 0 & 이 & 이 & $:$ & $\begin{array}{ll}1 & 1 \\
1\end{array}$ & $\begin{array}{ll}1 & 1\end{array}$ & 11 & \\
\hline$\overline{\mathbf{y}}$ & $\frac{0}{i s}$ & - & $\varphi$ & n & $i$ & $\begin{array}{ll}8 & \circ \\
10\end{array}$ & 요 & 용 & \\
\hline 흥 & \&) & 이 & o & o & 1) & $\begin{array}{ll}1 & 1\end{array}$ & $\begin{array}{ll}1 & 1\end{array}$ & $i$ & \\
\hline 입 & 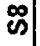 & 이 & o & o & : & $1:$ & $\begin{array}{ll}1 & 1\end{array}$ & $1 \quad 1$ & \\
\hline 点 & $\tilde{\omega}$ & 이 & o) & 0 & $:$ & $1 \quad:$ & $\begin{array}{ll}1 & 1\end{array}$ & $1 \quad:$ & \\
\hline 宓 & $\stackrel{\mathscr{~}}{\mathscr{6}}$ & o) & o & 0 & i) & $1 \quad 1$ & $: \quad:$ & $1: 1$ & \\
\hline$\stackrel{\alpha}{a}$ & (5) & - & $\infty$ & $\nabla$ & 1 & 8 잉 & $8 \mathrm{~B}$ & 옹 & \\
\hline & $\dot{~}$ & - & $\infty$ & 10 & 1 & $8 ㅇ$ & 읏 요 & 요 & \\
\hline & $\mathscr{B}$ & 0 & o & o & $i$ & 11 & $\begin{array}{ll}1 & 1\end{array}$ & $\begin{array}{ll}1 & 1\end{array}$ & \\
\hline 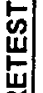 & ภै & - & $N$ & $M$ & ल) & 으 & ㅇ 8 & ᄋ & \\
\hline & s) & - & क) & $\infty$ & $:$ & $\begin{array}{ll}1 & i\end{array}$ & $\begin{array}{ll}1 & 1\end{array}$ & $: \quad 1$ & \\
\hline & 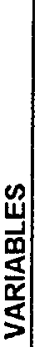 & 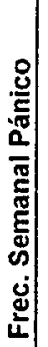 & 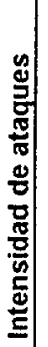 & 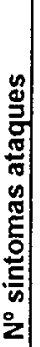 & 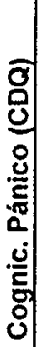 &  & 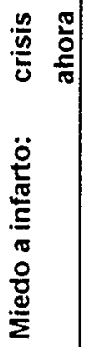 & 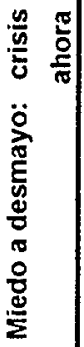 & 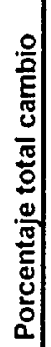 \\
\hline
\end{tabular}

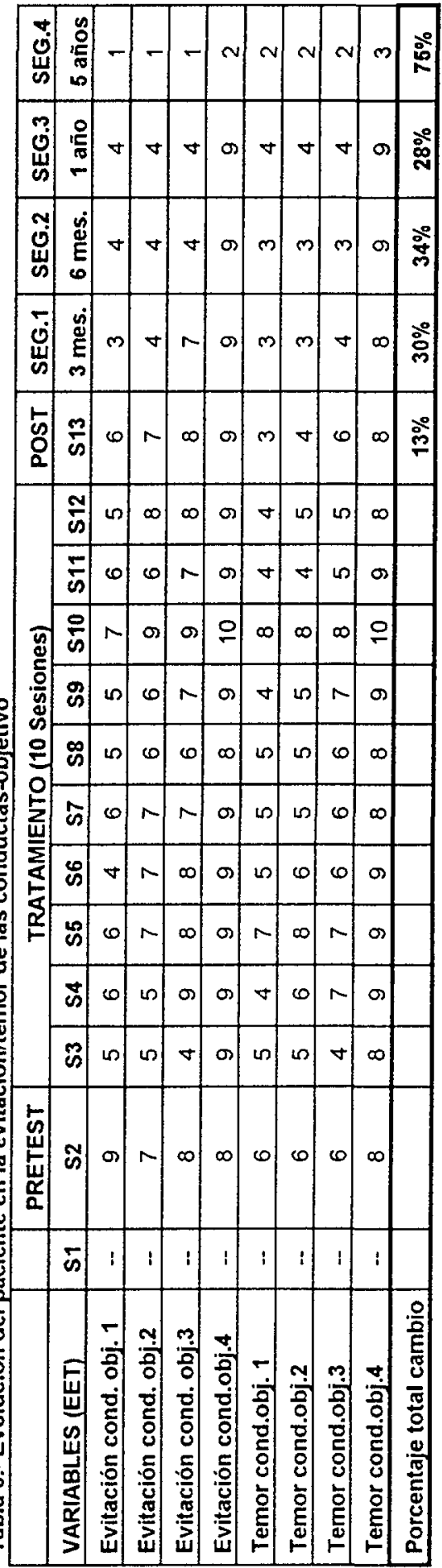



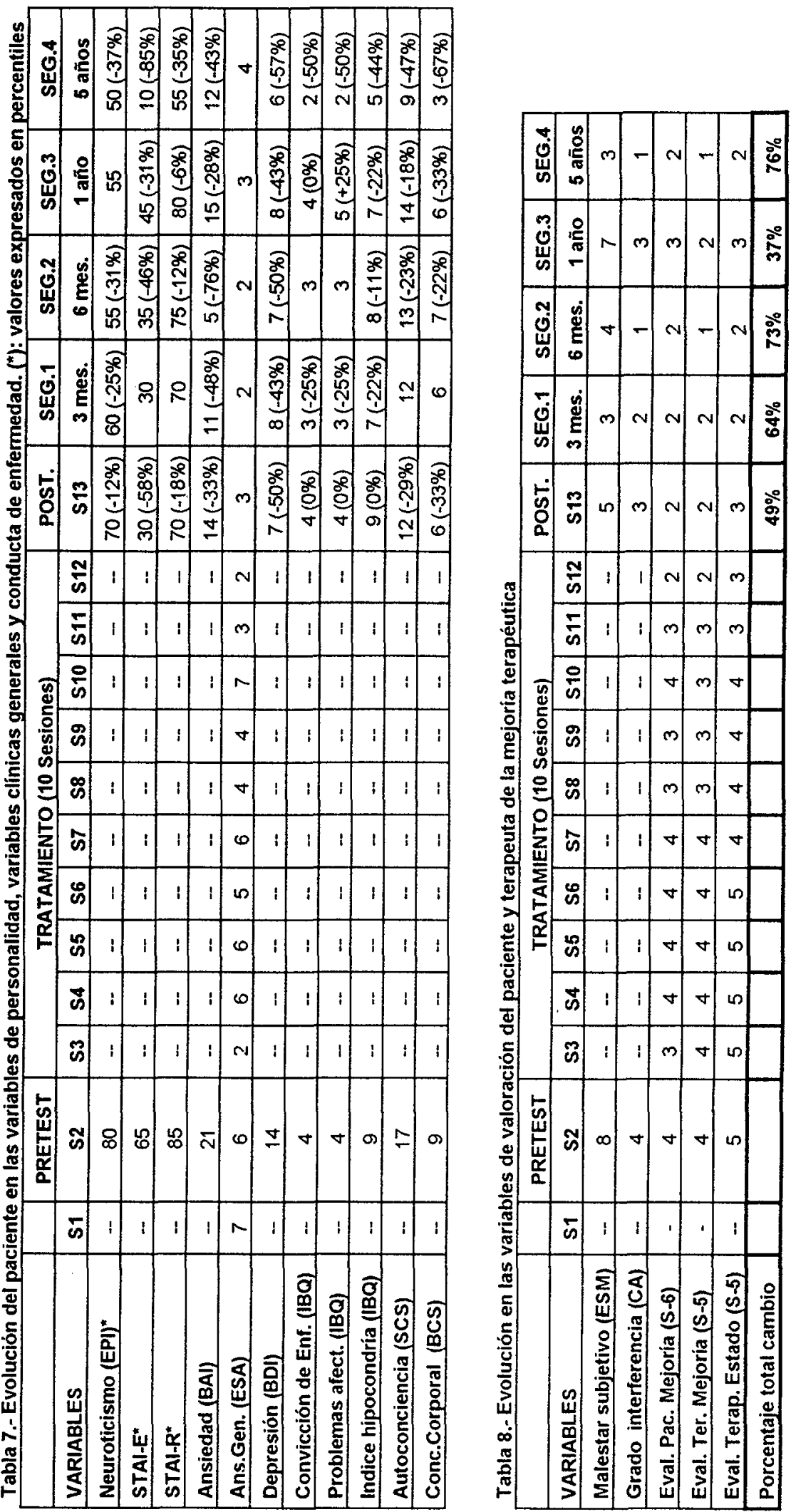
Figura 1.- Evolución del número de crisis de angustia a lo largo del proceso terapéutico

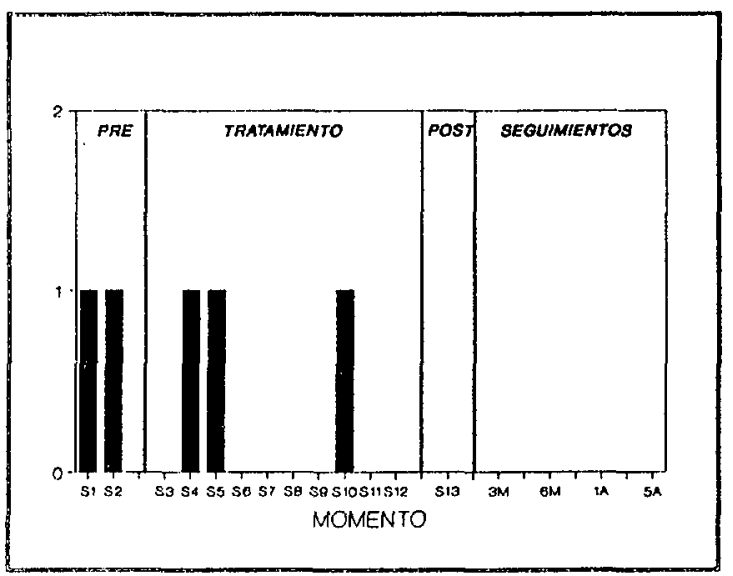

Figura 2.- Grado de creencia en cogniciones catastróficas a la largo del tratamiento

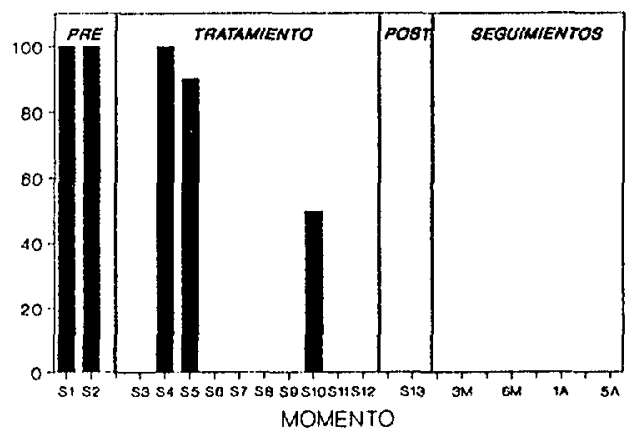

Figura 3.- Evolución en el grado de evitación-temor agorafóbicos para las conductas-objetivo

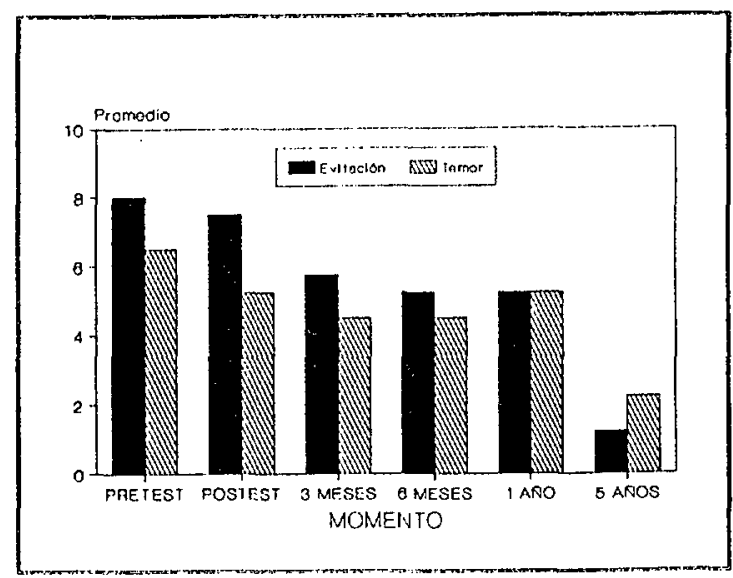

\subsection{Pretest (Semanas 1 y 2)}

En las tablas número 5, 6, 7 y 8 es posible ver los datos relativos a las diferentes variables en el pretest, esto es, antes de ninguna intervención. Como se puede ver, dentro de las variables directamente relacionadas con las crisis de angustia, J.V. informó haber sufrido 4 crisis en el último mes, de las cuales 1 había ocurrido esa semana, con una intensidad media de 7, un número de sensaciones corporales de 7, y una puntuación en el CDQ de 37. J.V. manifestó que las principales cogniciones catastróficas en las crisis eran "miedo a un ataque cerebral" (el grado de creencia en el momento de la crisis era del $100 \%$ y en el momento de la sesión, del $90 \%$ ), "miedo a un infarto" ( $70 \%$ en la crisis y $60 \%$ en la sesión) y "miedo al desmayo" ( $95 \%$ en crisis y $90 \%$ en sesión). Paciente y terapeuta fijaron como conductas-objetivo las de pasear, ir al cine, coger el autobús e ir de compras a un centro comercial, que presentaban unos niveles de evitación de $9,7,8$ y 8 respectivamente y un grado de temor de 6,6,6 y 8 respectivamente. Respecto a otras variables de personalidad, clínicas y de conducta de enfermedad, J.V. obtuvo una puntuación centil de 80 en el factor de neuroticismo del EPI, centil 65 en Ansie- 
dad-estado, centil 85 en Ansiedad-rasgo, 21 en el Inventario de Ansiedad de Beck (BAI) y 14 en el Inventario de depresión de Beck ${ }^{3}$. Dentro de los aspectos de conducta de enfermedad del paciente, éste obtuvo una puntuación de 4 en el factor de Convicción de Enfermedad, 4 en Problemas afectivos y 9 en el Indice Whiteley de Hipocondría del $1 \mathrm{BQ}^{4}$. Asimismo obtuvo una puntuación de 17 en el factor de Autoconciencia Privada (SCS) y de 9 en el de Conciencia corporal privada (BCS). Por lo que respecta a las variables de valoración de paciente y terapeuta, J.V. valoró con un 8 el malestar subjetivo experimentado por las crisis (ESM) y "mucho"4 el grado de interferencia global en su vida (CA). Tomando en consideración todas estas variables, el terapeuta valoró al paciente en el instrumento S-5 como "severamente perturbado"5 por su trastorno.

\subsection{Fase de Tratamiento 1 (Semanas 3 a 12)}

Por lo que respecta a la frecuencia de las crisis, a lo largo del tratamiento hubo semanas en las que el paciente no informó de ninguna crisis, algo que no le ocurria desde hacia mucho tiempo. Nuestro paciente experimentó tres crisis a lo largo de todo este periodo, concretamente en las semanas 4,5 y 10. Cabe destacar que la intensidad, así como las sensaciones corporales presentes en las crisis muestran una tendencia descendente 6 y 5 , en la última crisis). $Y$ algo similar ocurre con el grado de creencia de las principales cogniciones, que empieza a disminuir desde la segunda sesión de tratamiento y se sitúa casi en la mitad en la octava sesión de tratamiento. Por lo que respecta a la evitación/ temor agorafóbicos, a lo largo del tratamiento no hubo variaciones importantes. Más bien al contrario, el paciente presentó una importante resistencia a realizar experimentos conductuales que supusieran el incremento de su activación fisiológica y prácticamente no llevó a cabo ningún afrontamiento para poner a prue- ba las técnicas aprendidas y la veracidad de sus cogniciones por miedo a experimentar una crisis. Dentro del grupo de variables clínicas generales, el grado de ansiedad general experimentado durante la semana (ESA) tuvo una progresión ligeramente descendente. Por último, respecto a la valoración del paciente y del terapeuta, decir que el paciente informó en el S-6 sentirse "algo mejor" (3) ya en la primera sesión de tratamiento, aunque esta valoración presentó fluctuaciones hasta la sesión 8 , en que la progresión es más clara, evaluándose en la sesión 10 como "bastante mejor" (2). El terapeuta en el S-5 comienza a valorar al paciente como "algo mejor" (3) en la sesión 6 y "bastante mejor" en la sesión 10. Asímismo, la valoración del estado del paciente en la sesión 10 ya es de "moderadamente perturbado" (3).

Figura 4.- Evolución en el grado de hipocondria (Conducta de Enfermedad) del paciente

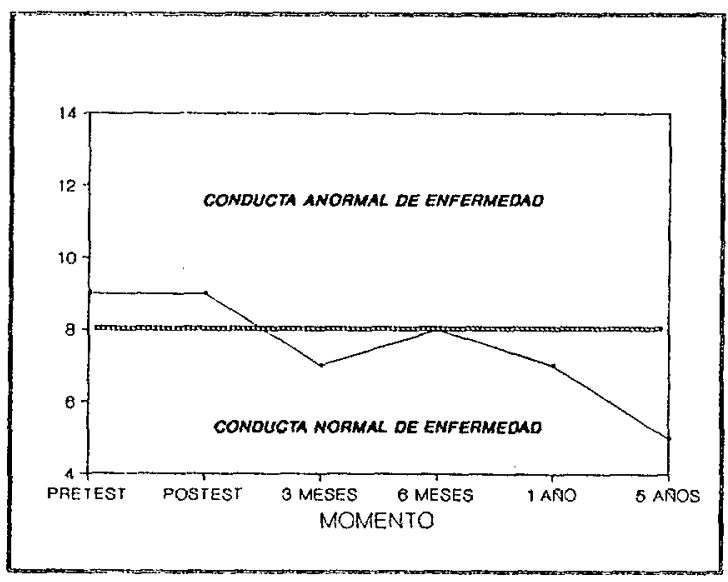

\footnotetext{
4 Un 10.9 se considera depresión ausente o minima en la versión de Conde y Franch, un 18.7 supone una depresión leve, un 25.5 depresión moderada y 30 es considerado como una depresión grave.

5 Se considera una conducta anormal de enfermedad en el factor Convicción de Enfermedad una puntuación entre 3 y 6 , y en el Indice Whiteley de Hipocondría, entre 8 y 14.
} 


\section{Tabla 9.- Contestaciones del paciente al "Examen sobre las crisis de angustia"}

\section{1) ¿Qué es una crisis de angustia y cómo se produce?}

"Una crisis es un estado que se caracteriza por una serie de sintomas fisicos importantes que padece el individuo y que le llevan a pensar que su muerte es inminente. Se produce por un círculo de pensamientos negativos, los cuales producen unos sintomas de ansiedad, mareo, etc. Dichos sintomas se interpretan catastróficamente aumentando los sintomas que, a su vez, generan más pensamientos negativos; éstos crean más sintomas y asi sucesivamente hasta llegar al momento del pánico".

2) Pensamientos que tengo ante una crisis de angustia. Evidencias a favor y en contra de la veracidad de tal pensamiento. Elementos de la terapia que me han resultado especialmente útiles,

\begin{tabular}{|c|c|c|}
\hline Pensamientos & Evidencias a favor & Evidencias en contra \\
\hline $\begin{array}{l}\text {-Voy a morir. } \\
\text { - Me puedo desmayar. } \\
\text { - Voy a tener un ataque cerebral. } \\
\text { - Esto es un ataque cardiaco. } \\
\text { - Mi cabeza va a explotar. } \\
\text {-Tengo mucho miedo. }\end{array}$ & $\begin{array}{l}\text { - Me encuentro muy mal. } \\
\text {-Estoy muy mareado. } \\
\text { - Mi cuerpo no funciona bien. } \\
\text { - No controlo mi cuerpo } \\
\text { (náuseas, ansiedad, } \\
\text { inestabilidad, falta de aire...). }\end{array}$ & $\begin{array}{l}\text {-La ansiedad es una respuesta } \\
\text { normal del organismo. } \\
\text {-Todo lo producen mis pensamientos. } \\
\text {-Si me analizo en este momento, realmente } \\
\text { no ocurre nada importante. } \\
\text {-Soy joven y estoy sano. } \\
\text {-Las crisis de angustia no son peligrosas. } \\
\text {-Es imposible desmayarse a causa de una } \\
\text { crisis. } \\
\text {-Esto me ha pasado ya muchas veces y } \\
\text { nunca ocurre nada catastrófico. } \\
\text {-Soy capaz cuanto menos de retrasar las } \\
\text { crisis o hacer disminuir los sintomas sólo } \\
\text { desfocalizando el pensamiento. }\end{array}$ \\
\hline
\end{tabular}

Los elementos terapéuticos que me han resultado útiles son:

a) "Confirmar el diagnóstico de mi problema".

b) "Saber que no puedo desmayarme".

c) "Conocer el papel de la hiperventilación y aprender a respirar lentamente".

d) "Utilizar las técnicas de distracción".

e) "Poder contactar con el terapeuta con cierta facilidad".

3) ¿Cómo puedo afrontar una crisis de angustia?.

"En primer lugar, tratando de no asustarme demasiado y siendo consciente de que lo que me ocurre es única y exclusivamente una crisis de angustia. Aplicar una técnica o más de distracción, en cuanto sea posible practicar la respiración lenta y aprovechar la ligera mejoria para pesar con lógica y racionalmente".

Del curso del tratamiento sólo destacar la desconfianza del paciente respecto a su mejoría terapéutica, a pesar de su sorpresa por no experimentar crisis algunas semanas y su miedo hipervigilante a cualquier sensación corporal que perturbase el frágil equilibrio somático y emocional en el que se habia acostumbrado a vivir. También es notable que J.V. ya contaba con bastante información acerca del trastorno por angustia en el momento de acudir a consulta, información que no le servia de nada a la hora de controlar las crisis, puesto que permanecia un grado de creencia considerable en la posibilidad de consecuencias catastróficas en sus crisis. Su buen nivel intelectual facilitaba las discusiones cognitivas 
y su grado de comprensión en las sesiones. Una muestra de ello es la tarea que realizó en el "examen sobre las crisis de angustia" y que presentamos detalladamente en la tabla número 9. Sin embargo, la antigüedad del problema hacía que sus creencias erróneas estuviesen muy consolidadas y que su ansiedad se hallase fuertemente condicionada a multitud de estímulos internos y externos. Por otra parte, la atribución externa de los éxitos en el control de las crisis constituyó un factor muy perturbador a lo largo del tratamiento, de acuerdo con 10 indicado en algunos trabajos (Borden, Plum y Salmon, 1991).

\subsection{Postest (Semana 13)}

Como puede apreciarse en las tablas 5 a 8 , respecto a las variables directamente relacionadas con el pánico, la frecuencia de crisis de angustia en la última semana tras el tratamiento, asi como en el último mes, fue nula y la puntuación en el CDQ se redujo en un $27 \%$. Asímismo el grado de creencia en las principales interpretaciones catastróficas se habian reducido sustancialmente quedando en un $5 \%$ para el miedo a un ataque cerebral, un $3 \%$ para el miedo al infarto y un $5 \%$ para el miedo al desmayo. Estas puntuaciones reflejan una disminución cercana al $95 \%$ en el grado de creencia de los principales miedos. El porcentaje total de cambio respecto a las variables directamente relacionadas con las crisis se sitúa, pues, en un $98 \%$, lo que permite decir que el paciente, tras la primera fase de tratamiento se encontraba muy mejorado respecto a estas variables ${ }^{\hat{\sigma}}$.

Otro comentario muy distinto merece el análisis de los datos referidos a la evitación/temor agorafóbicos (EET) que apenas reflejan una disminución global del $13 \%$, esto es, nula mejoría.

Por lo que se refiere a las variables de personalidad, clinicas y de conducta de enfermedad, J.V. obtuvo tras el tratamiento una puntuación centil de 70 en el factor de neuroticismo del EPI, centil 30 de Ansiedad-estado, centil
70 de Ansiedad-rasgo, 14 en el Inventario de Ansiedad de Beck (BAI), 3 en la Ansiedad general de la semana (ESA) y 7 en el Inventario de depresión de Beck (BDI), por lo que se aprecia una ligera disminución en todas estas variables. Dentro de los aspectos de conducta de enfermedad del paciente, ésta no varía en absoluto en los factores de Convicción de Enfermedad e Indice Whiteley de Hipocondría del IBQ. Asimismo obtiene una puntuación ligeramente mejorada de 12 en el factor de Autoconciencia Privada (SCS) y de 6 en el de Conciencia corporal privada (BCS).

Las variables de valoración del paciente y terapeuta experimentaron una disminución del $49 \%$, esto es, J.V. mejoró de modo importante tras el tratamiento si tenemos en cuenta estas variables. Más concretamente, el paciente valoró con un 5 el malestar subjetivo experimentado a causa de su problema (ESM) y "bastante" (3) el grado de interferencia global en su vida (CA). Asímismo el paciente se calificó como "bastante mejor" (2) comparándose con el inicio del tratamiento (S-6). Tomando en consideración todas estas variables, el terapeuta valoró al paciente en el S-5 como "moderadamente perturbado" (3), encontrándolo "bastante mejor" (2) en ese momento que antes del tratamiento.

En resumen, si seguimos la clasificación de Visser y Bouman (1992), es posible afirmar que tras el tratamiento 1 , nuestro paciente se encontraba muy mejorado respecto a las variables directamente relacionadas con las crisis de angustia, mejorado respecto a las variables de valoración global de paciente y terapeuta y sin cambios respecto a las variables de evitación-temor agorafóbicos y las variables de personalidad, clínicas generales y de conducta de enfermedad.

\subsection{Primer seguimiento ( 3 meses)}

En el comentario de la información recogida en el primer seguimiento solamente resaltaremos los datos más importantes, especialmente los que varien de forma significa-

\footnotetext{
${ }^{6}$ No tenemos en cuenta en el promedio el dato referido al CDQ ya que, por el formato del cuestionario, se pregunta al paciente por las cogniciones presentes en las crisis. Cuando no hay crisis, el paciente en ocasiones contesta pensando en las últimas crisis que tuvo, por lo que consideramos que es una mala medida de cambio.
} 
tiva respecto al postest. Asi, podemos señalar que a los tres meses de finalizada la terapia se mantiene prácticamente idéntica la mejoría alcanzada con el tratamiento en las variables directamente relacionadas con las crisis, con un porcentaje de cambio del $98 \%$. Como dato a destacar, decir que en este intervalo de tiempo, el paciente no tuvo ni una sola crisis de angustia. La evitación-temor agorafobicos (EET) respecto a las conductas objetivo evolucionan en el sentido de una mejoria, alcanzando un porcentaje de cambio del $30 \%$, que ya denota una mejoria ligera a este respecto. Más concretamente disminuye la evitación para las conductas número 1 (pasear) y para las conductas 2 (ir al cine) y 3 (coger el autobús), que no se habian modificado en el postest y se mantiene idéntica la evitación de la conducta 4 (ir a un centro comercial). Por 10 que se refiere a las variables de personalidad, clínicas $y$ de conducta de enfermedad, se incrementa el porcentaje global de cambio, lo que ya permite hablar de una mejoria del paciente en estas variables. En este sentido, el paciente se sitúa en el centil 60 de neuroticismo, puntúa 11 en la ansiedad evaluada con el BAI y 2 en la Escala subjetiva de ansiedad (ESA). Aparecen por primera vez cambios en las variables de Convicción de Enfermedad (3), Problemas afectivos (3) e Hipocondría (7). Se mantiene la mejoria alcanzada en las variables Ansiedad-estado, Ansiedad-rasgo, Autoconciencia y Conciencia Corporal Privada. $Y$ en Depresión obtiene una puntuación ligeramente más elevada a la que obtuvo en la última evaluación. Por último, por lo que se refiere a las variables de valoración del paciente y terapeuta, se incrementa notablemente la mejoría, alcanzando un porcentaje global del $64 \%$. Se mantienen la valoración de paciente y terapeuta respecto al grado de mejoria y disminuye el malestar subjetivo (3) en la ESM, asi como el grado de interferencia (2) (CA), valorando el terapeuta al paciente como "ligeramente perturbado" (2) en el S-5.

En resumen, en el primer seguimiento realizado a los tres meses de finalizada la terapia, se mantiene la mejoría alcanzada en las variables directamente relacionadas con las crisis de angustia, observándose cambios ligeros en otras variables que hasta el momento no habian mejorado.

\subsection{Segundo seguimiento (6 meses)}

Del segundo seguimiento, realizado a los 6 meses de finalizada la terapia, cabe destacar de nuevo la ausencia de crisis de angustia, se mantiene la puntuación del CDQ y se incrementa muy ligeramente el grado de creencia asociado a las cogniciones catastróficas, quedando en $10 \%$ para el miedo al ataque cerebral, $5 \%$ para el miedo al infarto y $10 \%$ para el miedo al desmayo. El porcentaje global de cambio para las variables directamente relacionadas con las crisis queda prácticamente idéntico, situándose en el $97 \%$. En cuanto a la evitación-temor agorafobicos (EET), se incrementa muy ligeramente el nivel de mejoría alcanzado (disminución del $34 \%$ ), especialmente por lo que se refiere a la conducta-objetivo número 3 . El cambio global en las variables de personalidad, variables clínicas generales $y$ de conducta de enfermedad, se mantiene prácticamente igual. Disminuye la puntuación en Neuroticismo (centil 55), la ansiedad medida con el BAl (5) y la Depresión (7). Se mantiene la mejoría en las variables Ansiedad Semanal (ESA), Convicción de Enfermedad y Problemas Afectivos (IBQ). $Y$ se produce un ligero empeoramiento respecto a la última evaluación en las variables Ansiedad-Estado (centil 35), Ansiedad-Rasgo (centil 75), Hipocondria (8), Autoconciencia Privada (13) y Conciencia Corporal Privada (7). Por último, decir respecto a la valoración de paciente y terapeuta, que en el segundo seguimiento sigue aumentando de forma notable la mejoría alcanzada con el tratamiento $(73 \%)$, permitiendo afirmar que el paciente se encuentra muy mejorado si atendemos a la valoración de paciente y terapeuta. Así, disminuye el grado de interferencia (CA) creado por el problema (1), y el terapeuta valora al paciente en el S-5 como "muy mejorado" (1). Se mantiene la valoración del paciente acerca de su mejoría y la del terapeuta acerca del estado del paciente. $Y$ se produce cierto empeoramiento respecto a la última evaluación en cuanto al malestar subjetivo evaluado con la ESM (4\%).

En resumen, en el segundo seguimiento, se puede afirmar que sigue la evolución favorable observada en el primer seguimiento, esto es, sigue manteniéndose la importantísima mejoría en las variables directamente relacio- 
nadas con las crisis; también se mantiene la ligera mejoría obtenida en la evitación-temor agorafóbicos y en las variables clínicas generales; y se incrementa la mejoria en la valoración del terapeuta y paciente respecto del estado de éste último.

\subsection{Tercer seguimiento ( 1 año)}

Por lo que respecta a las variables directamente relacionadas con las crisis, el dato más notable al año de finalizar la terapia es que J.V. sigue sin crisis de angustia, a pesar de que el grado de creencia en los principales miedos se incrementa con respecto al segundo seguimiento. Asi, se observa una puntuación de 32 en el CDQ, $30 \%$ en el grado de creencia en relación al miedo al ataque cerebral, $20 \%$ en el miedo al infarto y $25 \%$ en el miedo al desmayo. El porcentaje total de cambio, se mantiene, no obstante, en niveles similares; concretamente en el $91 \%$, lo que nos indica que el paciente sigue muy mejorado con respecto a estas variables. La evitación-temor agorafóbicos (EET) empeora también respecto al segundo seguimiento, reduciéndose el porcentaje de cambio al $28 \%$, estó es, volvemos a hablar de una casi nula mejoría con respecto al pretest. Algo similar cabe decir de las variables de personalidad, variables clinicas generales y variables de conducta de enfermedad. Entre estas variables, sólo se incrementa la mejoria con respecto al segundo seguimiento en Hipocondría (7) y en Conciencia corporal privada (6). Se mantiene la puntuación obtenida en neuroticismo, y todas las restantes aumentan respecto al seguimiento anterior, a pesar de seguir mostrando una mejoria muy ligera con respecto al pretratamiento. Por último, cabe decir que en las variables de valoracion de paciente y terapeuta, se produce un importante empeoramiento con respecto al segundo seguimiento. Así, si en el segundo seguimiento hablábamos de un porcentaje de mejoría del $73 \%$, ahora se ve reducido hasta el $37 \%$. Todas estas variables empeoran: el malestar subjetivo (ESM) que ocasiona el problema es cifrado en 7 , el grado de interferencia (CA) se eleva a 3, el paciente se evalúa en la S-6 simplemente como "algo mejor" (3) y el terapeuta valora al paciente en la S-5 como "bastante mejor" (2), siendo necesario volver a situarle en la categoría de "moderadamente perturbado" (3) como en el postest.

En resumen, al año de finalizar la terapia se observa un empeoramiento del paciente respecto al segundo seguimiento en todas las variables. En este sentido, son las variables directamente relacionadas con las crisis las que menos acusan el empeoramiento, manteniendo prácticamente igual el nivel de mejoría del paciente en cuanto a frecuencia y cogniciones de pánico. Sin embargo, el empeoramiento en las variables de valoración subjetiva del paciente y valoración del terapeuta es importante. $Y$, por lo que respecta a las restantes variables, en las que el cambio apreciado hasta el momento era más tenue, también se ven ligeramente afectadas por este empeoramiento del paciente.

\section{7. Tratamiento $2(3,5$ años a 5 años) $(40$ sesiones)}

El empeoramiento de nuestro paciente en algunas variables clínicas (ansiedad-estado, ansiedad-rasgo, depresión, convicción de enfermedad, problemas afectivos, autoconciencia, malestar subjetivo, grado de interferencia del problema), asi como su estancamiento en la leve mejoría alcanzada con respecto a la evitación-temor agorafóbicos al año de finalizar la terapia, nos hizo temer que la evolución clínica del paciente siguiese a partir de entonces una trayectoria poco favorable. Sin embargo, aunque resultaba fácil de ver que el tratamiento 1 no había sido capaz de alterar significativamente esas variables, nos encontrábamos con que sí habían mejorado sustancialmente las variables directamente relacionadas con las crisis de angustia (frecuencia de crisis y cogniciones catastróficas) y que se mantenia la mejoria lograda en dos variables de conducta de enfermedad que se han revelado centrales en el trastorno por angustia, a saber, la hipocondría y la conciencia corporal privada (Ballester, 1992; Ballester y Botella, 1993a, b).

No se intervino hasta dos años y medio más tarde, cuando el paciente buscó de nuevo nuestra ayuda psicológica. Las razones de la 
renovada motivación del paciente que le llevaba a buscar de nuevo ayuda, ya han sido expuestas anteriormente, asi como el contenido de esta segunda fase del tratamiento. Lo importante a destacar aqui, es que el paciente seguia sin haber tenido ni una sola crisis completa desde la finalización del primer tratamiento. Por lo que respecta al tiempo en que se aplicó este segundo tratamiento, tampoco se dieron crisis, a pesar de que se llevaron a cabo exposiciones a estímulos (tanto internos como externos) muy temidos por el paciente. Más bien al contrario, se produjeron hechos muy significativos desde el punto de vista clínico, como por ejemplo, que J.V. manifestara en varias ocasiones (aproximadamente a los 4 . meses de haber iniciado esta segunda fase de tratamiento) que por vez primera en su vida había tenido la sensación de haber sido él mismo (con la puesta en marcha de las estrategias aprendidas) quien habia conseguido evitar una crisis. Esto nos mostraba que se habia comenzado a alterar la autoeficacia percibida en nuestro paciente, un elemento importante para el cambio terapéutico (Borden y cols, 1991). Otro hito digno de mención fue cuando al año de comenzar esta segunda fase de tratamiento, el paciente decidió inscribirse en un curso de tenis, sobre todo, teniendo en cuenta que el paciente no practicaba ningún ejercicio físico (evitaba cualquier esfuerzo) desde antes de comenzar con su problema, esto es, desde hacia 14 años y que el miedo a las sensaciones físicas constituye un importante mediador en las crisis de angustia (Rapee y Medoro, 1994). Como estudiosos interesados en la eficacia diferencial de los distintos componentes en el tratamiento del trastorno por angustia, también nos resultó sugerente el comentario del paciente al analizar las razones por las que iba incrementando su afrontamiento de situaciones antes evitadas: "En lo que se refiere a mis miedos frente a algunas situaciones, exponerme a ellas me ayuda más que todos los razonamientos de que soy capaz". Así, además de los datos referidos a las conductas objetivo que se comentarán más tarde, disminuyó la evitación y el temor agorafóbicos del paciente ante un gran número de situaciones como ir a distintos lugares sin la seguridad que le proporcionaba aparcar su coche en la puerta, viajar, ir a un restaurante, hacer colas, aglomeraciones de gente, tráfico, pasar calor, beber alcohol, disgustarse, realizar gestiones en organismos privados o públicos, emocionarse positivamente, ir solo con sus hijos y ducharse. Este importante cambio logrado al aplicar de una forma sistematizada la exposición a las situaciones temidas, nos habla de la importancia central de esta técnica en el tratamiento de la conducta de evitación. A continuación, detallamos los resultados que obtuvimos tras esta fase de tratamiento en el cuarto seguimiento.

\subsection{Cuarto seguimiento ( 5 años)}

Como comentario general acerca de los datos obtenidos a los cinco años de haber finalizado el tratamiento 1 y a los tres meses de finalizado el tratamiento 2, cabe destacar la importante mejoria que se registra en absolutamente todas las variables evaluadas. No sólo se recupera el grado de mejoría alcanzado en el segundo seguimiento, sino que se logran los niveles más altos alcanzados hasta el momento.

Si atendemos a las variables directamente relacionadas con las crisis, a los cinco años de haber finalizado el primer tratamiento el paciente no ha vuelto a experimentar ninguna crisis de angustia. Obtiene la puntuación más baja hasta la fecha en el CDQ (25), y el grado de creencia para todos los miedos se reduce al mínimo: $1 \%$ en el grado de creencia en relación al miedo al ataque cerebral, $0 \%$ en el miedo al infarto y $1 \%$ en el miedo al desmayo. Por tanto, el porcentaje total de cambio en las variables directamente implicadas en las crisis, se incrementa hasta constituir prácticamente el $100 \%$. La evitacion-temor agorafóbicos (EET) constituyen, sin duda, las variables más beneficiadas por el segundo tratamiento (que recuérdese incorporaba de forma sustancial el componente de exposición). El porcentaje total de cambio en la evitación y temor agorafóbicos aumenta hasta el $75 \%$ (los niveles de evitación no superan el nivel de 2 , y los de temor no superan el 3 para ninguna conducta objetivo). El paciente era capaz de ir a pasear, al cine, coger el autobús e ir a un centro comercial, aunque con cierta intranquilidad. También se observan cambios en las variables de personalidad, variables clínicas 
generales y variables de conducta de enfermedad. El paciente obtiene las puntuaciones más bajas hasta el momento en las variables de neuroticismo (puntuación centil 50), ansiedad-estado (10), ansiedad-rasgo (55), depresión (6), convicción de enfermedad (2), problemas afectivos (2), hipocondria (5), autoconciencia (9) y conciencia corporal privada (3). Por último, cabe decir que en las variables de valoración de paciente y terapeuta, se produce también una importante recuperación lográndose niveles de cambio algo superiores a los del segundo seguimiento $(76 \%)$. El malestar subjetivo causado por el problema (ESM) es valorado como un 3; el grado de interferencia (CA) llega a la calificación de "casi nada" (1); el paciente se evalúa de nuevo en la escala S-6 como "bastante mejor" (2); y el terapeuta valora por vez primera al paciente en la escala S-5 como "muchísimo mejor" (1), reservándose no obstante, la calificación de "ligeramente perturbado" (2) por su problema en el S-6. La razón de que el terapeuta no valorase al paciente como "normal" se debe a la permanencia, aunque con grado minimo, de ciertas conductas de evitación y de cierta hipervigilancia respecto al funcionamiento corporal, aspecto de gran relevancia en los pacientes con trastorno por angustia (Antony, Meadows, Brown y Barlow, 1994; Westling y Öst, 1995).

En resumen, a los cinco años de finalizar el primer tratamiento sigue manteniéndose la importante mejoria lograda con el primer tratamiento en las variables directamente relacionadas con las crisis y las de valoración del paciente y del terapeuta. $Y$, una vez aplicado el segundo tratamiento, por fin se logran cambios en las variables de evitación-temor agorafóbicos y variables clínicas generales que se habian mostrado insensibles al tratamiento inicial.

\section{CONCLUSIONES Y COMENTARIO FINAL}

A partir de los resultados presentados podemos extraer algunas conclusiones:

a) El programa cognitivo-comportamental estructurado en 10 sesiones, y especificamente desarrollado para el control de las crisis de angustia, resultó una alternativa terapéutica muy eficaz para el paciente, especialmente por lo que se refiere a los aspectos más centrales del trastorno, como son la frecuencia de las crisis y el grado de creencia de las cogniciones catastróficas. La conveniencia de su utilización queda resaltada si tenenos en cuenta su bajo coste (apenas diez sesiones) y la desaparición total de crisis de angustia incluso en un paciente que llevaba once años con este problema.

b) Nuestros resultados también permiten afirmar que la mejoría inicial alcanzada con este tratamiento en las variables directamente relacionadas con la manifestación de las crisis de angustia se mantiene e incluso se incrementa a largo plazo (en el presente caso, a lo largo de un período de 5 años).

c) Por lo que respecta a la generalización de los cambios conseguidos, hay que señalar que el programa estructurado en 10 sesiones aplicado a este paciente concreto, produce efectos de generalización en variables relevantes como por ejemplo, la ansiedad-estado, la depresión y variables de conducta de enfermedad tales como la hipocondria, autoconciencia y conciencia corporal privada, con una disminución considerable a lo largo de los distintos seguimientos previos a la aplicación del tratamiento 2. Estos últimos resultados concuerdan con los hallados en un trabajo previo (Ballester, 1992) en el que este mismo programa de tratamiento se mostró eficaz para producir cambios significativos en el grado de hipocondria y la conciencia corporal privada de una muestra compuesta por 19 pacientes. Más concretamente, en el caso de la hipocondría se produjo un descenso importante de la puntuación obtenida por todos los pacientes al final del tratamiento, y 7 de los 15 sujetos cuya puntuación superaba el punto de corte de la "conducta anormal de enfermedad" (puntuación mayor que 8) se vieron fuera de esta categoría al finalizar el programa, manteniéndose estos resultados en un seguimiento realizado seis meses después. Por otra parte, en el caso que presentamos, algunas variables como la ansiedad-rasgo y la convicción de enfermedad apenas se vieron modificadas por este tipo de intervención.

d) En esta misma línea cabe hablar de lo que ocurre con la evitación agorafóbica con la 
aplicación del tratamiento 1. El programa terapéutivo descrito, en el que, recordemos, el componente de exposición no se plantea de un modo estructurado y sistematizado, consigue alterar, aunque ligeramente, las conductas de evitación. En relación a este resultado queremos recordar un trabajo previo de Botella (1992) con una muestra de 16 pacientes con diagnóstico de trastorno por angustia. A estos pacientes se les aplicó este mismo programa de tratamiento de 10 sesiones y, a nivel de grupo, se produjeron cambios más importantes en la evitación y temor agorafóbicos que los observados en nuestro paciente. Bajo nuestro punto de vista, esa diferencia en cuanto al grado de cambio observado cabe explicarla a partir de la gravedad de la evitación y temor agorafóbicos. En el caso que aquí presentamos las conductas de evitación abarcaban un amplísimo espectro del funcionamiento cotidiano del paciente y se hallaban profundamente enraizadas. Ya resulta notable que, sin una exposición estructurada, la evitación y el temor agorafóbicos desciendan. Ahora bien, dada la gravedad de estos factores en nuestro paciente, parece necesario recurrir a algo muy sistemático para lograr una mejoría clínicamente significativa. No resulta muy extraño, por tanto, que la evitación y temor agorafóbicos mejoraran tras la aplicación del tratamiento 2 que incorpora de modo estructurado el componente de exposición, resultado éste que se sitúa en la línea de otros trabajos como el de van Den Hout, Arntz y Hoestra (1994).

e) Además de conseguir que las conductas de evitación se vean alteradas de modo significativo, el tratamiento 2 produce efectos mucho más potentes que el tratamiento 1 en otras variables clínicas no directamente relacionadas con la manifestación de las crisis tales como la ansiedad-estado y ansiedad-rasgo y las variables relacionadas con la conducta de enfermedad del paciente tales como su convicción de enfermedad, el grado de hipocondría y la conciencia corporal privada.

Ahora bien, hay que decir que, además de los efectos beneficiosos del componente de exposición para la evitación agorafóbica, otro posible factor explicativo de que las conductas de evitación, así como la conducta de enfermedad de nuestro paciente se hayan visto alteradas de modo mucho más significativo con el tratamiento 2 , reside en el hecho de que que éste es bastante más prolongado. Podemos hipotetizar, por tanto, que un tratamiento más largo, en el que se da un mayor contacto terapéutico y se ofrece al paciente una mayor oportunidad para trabajar las técnicas de control de las crisis de angustia, aumenta la posibilidad de lograr cambios significativos en distintos tipos de variables. De hecho, en el caso que presentamos estos cambios se producen.

Sin embargo, no pensamos que esa sola sea la respuesta, sino que deberíamos seguir planteándonos posibles explicaciones del cambio: ¿qué factor o factores son los responsables del mayor alcance terapéutico logrado con un tratamiento más prolongado?. Como acabamos de señalar, ¿se trata únicamente de una cuestión temporal, esto es, del tiempo necesario para la consolidación del aprendizaje de nuevas habilidades?. ¿Implica ello que en los casos difíciles habrá que aplicar las mismas técnicas de control de las crisis un mayor número de veces?. ¿Estas técnicas serán igualmente eficaces si se aplican reduciendo al minimo el contacto con el terapeuta? (Coté, Gauthier, Laberge, Cormier y Plamondon, 1994). Y, en esta misma línea, ¿se puede entrenar a los pacientes en el control de las crisis simplemente a través del uso de biblioterapia con los mismos niveles de eficacia? (Gould, Clum y Shapiro, 1993). Por tanto, ¿es fundamentalmente el trabajo del paciente el factor responsable del cambio y, practicamente, se podría prescindir del terapeuta?. ¿O la explicación es otra, y lo que resulta necesario lograr (con más o menos tiempo) es que el paciente perciba control sobre sus crisis alcanzando la sensación de autoeficacia percibida que, según algunos autores (Borden, Clum y Salmon, 1991; Rapee, 1993), resulta central en el trastorno por angustia? ¿O acaso el factor fundamental que deberiamos conseguir alterar es la hipervigilancia somática y los sesgos cognitivos relacionados con la amenaza física? (Steptoe y Vögele, 1992; Rappe y Medoro, 1994; Anthony y cols. 1994; Ehlers, Breuer, Dohn y Fiegenbaum, 1995; Westling y Öst, 1995). ¿O en el caso de la evitación agorafóbica, es necesario eliminar las restric- 
ciones percibidas en la vida del sujeto? (Cox, Endler y Swinson, 1995).

Nos faltan respuestas exactas para tales cuestiones, pero intuimos que la mayor parte de los aspectos citados pueden resultar importantes. También pensamos que para poder alterar de modo significativo variables tan relevantes como las comentadas, seguramente, resulta necesario un tratamiento prolongado que incluya distintos componentes terapéuticos pensados para cada uno de esos aspectos (aunque sea posible esperar generalizaciones y, quizás, no fuera preciso abordar separadamente y de forma minuciosa cada uno de ellos). Lo que parece seguro es que en los casos más graves, como el que aqui presentamos, en los que el problema está muy cronificado y se ha instalado en la vida de la persona impregnado, prácticamente, todo su funcionamiento, habrá que apelar a tratamientos complejos, aplicados durante el suficiente tiempo como para que resulte posible eliminar las "capas patológicas" o "costras" que impiden al paciente vivir y funcionar como él desearia.

Por otra parte, un modo sensato y obvio a través del cual podemos intentar responder a algunas de las anteriores preguntas es seguir la evolución de los pacientes a lo largo de periodos de tiempo prolongados y con el máximo control respecto a una serie de variables que se hipotetiza pueden estar ejerciendo influencia en el cambio terapéutico y en su mantenimiento. En el caso que presentamos, hemos podido comprobar que se produjo un momento de inflexión y de empeoramiento en, prácticamente, todas las variables al año de finalizar la terapia. Este empeoramiento, hipotéticamente, habría podido dar lugar a una recaída y a la reaparición de las crisis de angustia. Nuestro conocimiento de este trastorno todavía no es lo suficientemente profundo como para poder predecir en qué pacientes se pueden producir estos "baches" y cuáles son los factores que determinarán, finalmente, que se produzca o no la recaída. Algunos trabajos como el recientemente publicado de Ehlers (1995) comienzan a atender estos aspectos.

$\mathrm{Ni}$ siquiera sabemos si resulta excesivamente ambicioso pretender o esperar que una persona que ha padecido alguna vez en su vida reacciones intensas de ansiedad deje de experimentarlas para siempre con cualquier tipo de tratamiento de cualquier duración? ${ }^{7}$. Conocer con profundidad los factores psicológicos que están en la base del trastomo por angustia nos ayudaría a preverlos y preverlos nos permitiría prevenirlos tomándolos en consideración a la hora de diseñar los tratamientos e incluso en campañas preventivas a nivel comunitario. Es prioritario, pues, seguir investigando.

\section{REFERENCIAS BIBLIOGRÁFICAS}

Antony, M.M., Meadows, E.A., Brown, T.A. yBarlow, D.H. (1994). Cardiac Awareness before and after cognitive-behavioral treatment for panic disorder. Journal of Anxiety-Disorders, vol 8, $\mathrm{n}^{\circ} 4, \mathrm{pp}$ 341-350.

American Psychiatric Association (1987). Manual Diagnóstico y Estadístico de los trastornos mentales revisado. (DSM-III-R). Masson, S.A. Barcelona.

Ballester, R. (1992). Análisis de los actuales planteamientos cognitivos acerca del trastorno de pánico y el concepto de conducta de enfermedad. Tesis Doctoral. Universidad de Valencia.

Ballester, R. y Botella, C. (1992). Aplicación del programa terapéutico de Clark a un caso con trastorno de pánico. Psicothema, vol.4, n², 6988.

Ballester, R. y Botella, C. (1993a). Perfil de conducta de enfermedad en pacientes con crisis de angustia. Análisis y Modificación de Conducta, vol. $19, n^{2} 64,151-304$

\footnotetext{
${ }^{7}$ En este sentido podemos decir que, apenas unos dias antes de la finalización de este trabajo, nuestro paciente nos telefone 6 para decirnos que estaba experimentando un nuevo brote de ansiedad como consecuencia de las obligaciones que le imponia el estar criando en su casa un cachorro de perro recién adquirido. Sin embargo, aunque el paciente manifestó estar muy alterado por este hecho (lo que muestra la persistencia de una cierta vulnerabilidad del paciente), también hay que decir que éste fue capaz de enfrentarse a la situación por si solo y controlar su reacción de ansiedad sin necesidad de acudir a terapia. Parece, por tanto, que J.V. habia aprendido a manejar su ansiedad a partir de nuestra intervención psicológica.
} 
Ballester, R. y Botella, C. (1993b). Autoconciencia y conciencia corporal en pacientes con trastorno por angustia. Análisis y Modificación de Conducta, vol. $19, n^{\circ} 67,613-642$.

Ballester, R., Botella, C. y Carrió, C. (1995). Eficacia diferencial del componente terapéutico cognitivo para el trastorno de pánico: análisis experimental de un caso. Análisis y Modificación de Conducta, vol.21, $\mathrm{n}^{\circ} 77$.

Barlow, D.H. (1988). Anxiety and its disorders. The nature and treatment of anxiety and panic. The Guilford Press.

Barlow, D.H. y Cerny, J.A. (1988). Psychological treatment of panic. The Guilford Press.

Beck, A.T. (1988). Cognitive approaches to panic disorder. Theory and therapy. En S. Rachman y J.D. Maser (comps.): Panic: Psychological perspectives. New Jersey, Lawrence Erlbaum Associates.

Beck, A.T., Emery, G. y Greenberg, R. (1985). Anxiety disorders and phobias: a cognitive perspective. New York. Basic Books.

Borden, J.W., Clum, G.A., Salmon, P.G. (1991). Mechanisms of change in the treatment of panic. Cognitive Therapy and Research, vol $15, n^{\circ} 4$, pp 257-272.

Botella, C. (1992). Trastorno por angustia. Manuscrito no publicado en Lectura de Cátedra. Universidad de Murcia.

Botella, C. y Ballester, R. (1991). Tratamiento psicológico del trastorno de pánico: adaptación del programa cognitivo-comportamental de Clark y Salkovskis. Análisis y Modificación de Conducta, 17, 871-894.

Clark, D. y Salkovskis, P. (1987). Cognitive treatment for panic attacks: therapist's manual. Manuscrito no publicado.

Clum, G.A. (1989). Psychological interventions versus drugs in the treatment of panic. Behavior Therapy, 20, 429-457.

Clum, G.A., Clum, G.A y Suris, R. (1993). A metaanalysis of treatments for panic disorder. Journal of Consulting and Clinical Psychology. 61, 2, 317-326.

Conde, V. y Franch, J.I. (1984). Escalas de evaluación comportamental para la cuantificación de la sintomatología psicopatológica en los trastornos angustiosos y depresivos.

Coté, G., Gauthier, J.G., Laberge, B., Cormier, H.J. y Plamondon, J. (1994), Reduced therapist contac in the cognitive behavioral treatment of panic disorder. Behavior Therapy, 25, 123-145.

Cox, B.J., Endler, N.S. y Swinson, R.P. (1995). An examination of levels of agoraphobic severity in panic disorder. Behavior Research Therapy, vol 33, $n^{\circ} 1$, pp 57-62.

Ehlers, A. (1995). A 1-year prospective study of panic attacks: clinical course and factors associated with maintenance. Journal of Abnormal Psychology, vol.104, ña, 164-172.

Ehlers, A., Breuer, P., Dohn, D. y Fiegenbaum, W. (1995). Heartbeat perception and panic disorder: possible explantions for descrepant findings. Behavior Research and Therapy, vol 33, $n^{\circ} 1$, pp 69-76.

Eysenck, H.J. y Eysenck, S.B. (1978). Cuestionario de Personalidad EPI. Madrid. TEA.

Fenigstein, A., Scheier, M.F. y Buss, A.H. (1975). Public and Private self-consciousness: assessment and theory. Journal of Consulting and Clinical Psychology, 43(4), 522-527.

Gould, R.A., Clum, G.A. y Shapiro, D. (1993). The use of bibliotherapy in the treatment of panic: a preliminary investigation. Behavior Therapy, 24, 241-252.

Ley, R. (1985). Agoraphobia, the panic attack and the hyperventilation syndrome. Behavioral Research and Therapy, 23, 79-81.

Ley, R. (1987). Panic disorder: A hyperventilation interpretation. En L.Michelson y L.M. Ascher (comps): Anxiety and stress disorders: cognitive behavioral assessment and treatment. New York. Guilford.

Ley, R. (1988). Hyperventilation and lactate infusion in the production of panic attacks. Clinical Psychology Review, 8, 1-18.

Lum, L.C. (1976). The syndrome of habitual chronic hyperventilation. En O.W. Hill (ed): Modern trends in Psychosomatic Medicine, vol.3. London. Butterworths.

Lum, L.C. (1981). Hyperventilation and anxiety state. J.R.Soc.Med., 74, 1-4.

Margrat, J., Barlow, D.H., Clark, D.M. y Tejch, M.J. (1993). Psychological treatment of panic: work in progress on outcome, active ingredients and follow-up. Behavioural Research and Therapy, $31,1,1-8$.

Marks, I.M. (1987). Fears, phobias and rituals. Oxford University Press. New York.

Martínez, M.P. y Botella, C. (en prensa). Aplicación de un tratamiento cognitivo-conductual a un caso de hipocondría primaria. Análisis y Modificación de Conducta.

Michelson, L.K. y Marchione, K. (1991). Behavioral, cognitive and pharmacological treatments of panic disorder with agoraphobia: critique and synthesis. Journal of Consulting and Clinical Psychology, 59, 1, 100-114.

Miller, L.C., Murphy, R. y Buss, A.H. (1981). Consciousness of body: private and public. Journal of Personality and Social Psychology, $41,2,397-406$.

Pilowsky, l. y Spence, N. (1983). Manual for the IIIness Behaviour Questionnaire. University of Adelaide. 
Rapee, R. (1993). Psychological factors in panic disorder. Adv. Behav. Res. Ther., vol 15, pp 85-102.

Rapee, R y Medoro, L. (1994). Fear of Physical sensations and trait anxiety as mediators of the response to hyperventilation in nonclinical subjects. Journal of Abnormal Psychology, vol. 103. $n^{\circ} 4,693-699$.

Salkovskis, P., Clark, D. y Hackman, A. (1991). Treatment of panic attacks using cognitive therapy without exposure or breathing retraining. Behaviour Research and Therapy.

Spielberger, C.D., Gorsuch, R.L. y Lushene, R.E. (1970). Manual for the state-trait anxiety inventory. Palo Alto. California. Consulting Psychologist Press.
Steptoe, A. y Vögele, C. (1992). Individual differences in the perception of bodily sensations: the role of trait anxiety and coping style. Behavior Research and Therapy, vol 30, $n^{\circ} 6$, pp 597-607.

Van den Hout, M., Arntz, A. y Hoekstra, R. (1994). Behaviour Research and Therapy, vol32, $n^{\circ} 4$, 447-451.

Visser, S. y Bouman P.K. (1992). Cognitivebehavioural approaches in the treatment of hypochondriasis: six-single case cross-over studies. Behaviour Research and Therapy, vol. 30 (3), 301-306.

Westling, B.E. y Ost, L.G. (1995). Cognitive bias in panic disorder patients and changes after cognitive-behavioral treatments. Behavior Research and Therapy, vol 33, no 5, pp 585-588. 